Erratum

\title{
Erratum to "Liproxstatin-1 Protects Hair Cell-Like HEI-OC1 Cells and Cochlear Hair Cells against Neomycin Ototoxicity"
}

\author{
Zhiwei Zheng, ${ }^{1,2}$ Dongmei Tang, ${ }^{1,2}$ Liping Zhao,, ${ }^{1,2}$ Wen Li, ${ }^{1,2}$ Jinghong Han, ${ }^{3}$ Bing Hu, ${ }^{3}$ \\ Guohui Nie, ${ }^{3}$ and Yingzi $\mathrm{He} \mathbb{i D}^{1,2}$ \\ ${ }^{1}$ ENT Institute and Department of Otorhinolaryngology, Eye \& ENT Hospital, Fudan University, Shanghai 200031, China \\ ${ }^{2}$ NHC Key Laboratory of Hearing Medicine (Fudan University), Shanghai 200031, China \\ ${ }^{3}$ Department of Otolaryngology and Institute of Translational Medicine, Shenzhen Second People's Hospital/The First Affiliated \\ Hospital of Shenzhen University Health Science Center, Shenzhen 518035, China \\ Correspondence should be addressed to Guohui Nie; nieguohui@email.szu.edu.cn and Yingzi He; yingzihe09611@126.com \\ Received 11 November 2021; Accepted 11 November 2021; Published 13 December 2021
}

Copyright (C) 2021 Zhiwei Zheng et al. This is an open access article distributed under the Creative Commons Attribution License, which permits unrestricted use, distribution, and reproduction in any medium, provided the original work is properly cited.

In the article titled "Liproxstatin-1 Protects Hair Cell-Like HEI-OC1 Cells and Cochlear Hair Cells against Neomycin Ototoxicity" [1], the incorrect figure files were introduced by the Editorial staff during the publication process. The figures should be corrected as follows: 


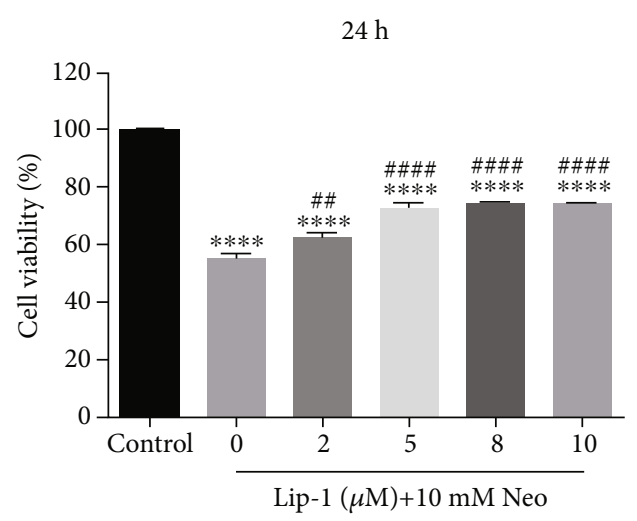

Figure 2: (d) Neomycin damaged cells were cotreated with or without Lip-1. Cell viability was measured by CCK8 kit. Values were represented as the mean \pm s.e.m. ${ }^{* *} p<0.01$ and ${ }^{* * * *} p<$

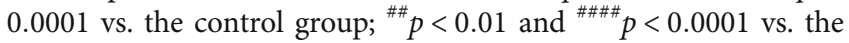
neomycin group.

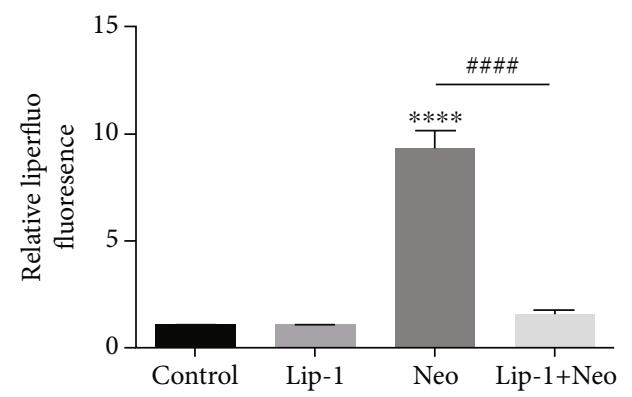

FIGURE 3: (j) Quantification of Liperfluo staining in HEI-OC1 cells confirmed a significant reduction with Lip-1 administration. Values were represented as the mean \pm s.e.m. ${ }^{* * * *} p<0.0001$ vs. the control group; ${ }^{\# \# \#} p<0.0001$ vs. the neomycin group, $n=6$.

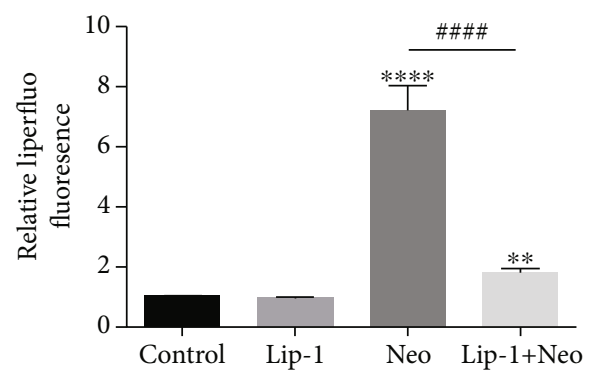

FIgURE 6: (j) Quantification of Liperfluo staining in cochlear hair cells confirmed a significant reduction with Lip-1 administration. Values were represented as the mean \pm s.e.m. ${ }^{* *} p<0.01$ and ${ }^{* * * *}$

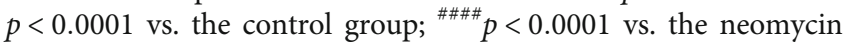
group, $n=10$.

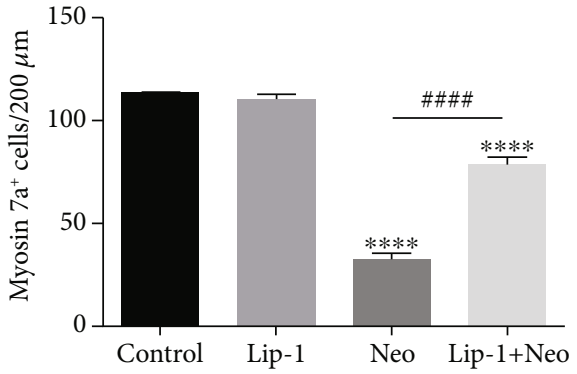

FIgURE 7: (e) Quantification of myosin 7a-positive hair cells in the middle turns of different groups. Values were represented as the mean \pm s.e.m. ${ }^{* * * *} p<0.0001$ vs. the control group; ${ }^{\# \# \#} p<0.0001$ vs. the neomycin group, $n=6$.
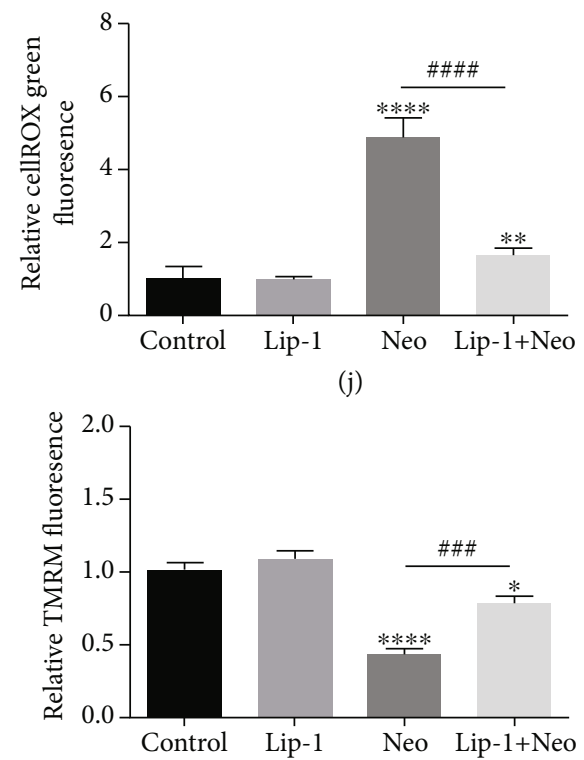

(o)

FIGURE 8: (j) Quantification of cellROX green staining in cochlear hair cells. (o) Quantification of TMRM staining in cochlear hair cells. Scale bars indicate $20 \mu \mathrm{m}$. Values were represented as the mean \pm s.e.m. ${ }^{*} p<0.05,{ }^{* *} p<0.01,{ }^{* * *} p<0.001$, and ${ }^{* * * *} p<$ 0.0001 vs. the control group; ${ }^{\# \#} p<0.001$ and ${ }^{\# \# \#} p<0.0001$ vs. the neomycin group, $n=10$.

\section{References}

[1] Z. Zheng, D. Tang, L. Zhao et al., "Liproxstatin-1 protects hair cell-like HEI-OC1 cells and cochlear hair cells against neomycin ototoxicity," Oxidative Medicine and Cellular Longevity, vol. 2020, Article ID 1782659, 15 pages, 2020. 